\title{
A SIMPLE PROOF OF A REMARKABLE CONTINUED FRACTION IDENTITY
}

\author{
P. G. ANDERSON, T. C. BROWN, AND P. J.-S. SHIUE
}

(Communicated by William W. Adams)

ABSTRACT. We give a simple proof of a generalization of the equality

$$
\sum_{n=1}^{\infty} \frac{1}{2^{[n / \tau]}}=\left[0,2^{0}, 2^{1}, 2^{1}, 2^{2}, 2^{3}, 2^{5}, \ldots\right],
$$

where $\tau=(1+\sqrt{5}) / 2$ and the exponents of the partial quotients are the Fibonacci numbers, and some closely related results.

\section{INTRODUCTION}

P. E. Böhmer [3], L. V. Danilov [4], and W. W. Adams and J. L. Davison [1] showed independently that if $\alpha>0$ is irrational, $b>1$ is an integer, and $S_{b}(\alpha)=(b-1) \sum_{k=1}^{\infty} \frac{1}{b^{k / \alpha]}}$, then the simple continued fraction for $S_{b}(\alpha)$ can be described explicitly in the following way. Let $\alpha$ have simple continued fraction

$$
\alpha=a_{0}+\frac{1}{a_{1}+\frac{1}{a_{2}+\cdots}}=\left[a_{0}, a_{1}, \ldots\right],
$$

with $\frac{p_{n}}{q_{n}}=\left[a_{0}, \ldots, a_{n}\right], n \geq 0$. Let $t_{0}=a_{0} b, t_{n}=\frac{b^{q_{n}}-b^{q_{n-2}}}{b^{q_{n-1}}-1}, n \geq 1$. Then $S_{b}(\alpha)=\left[t_{0}, t_{1}, \ldots\right]$. Thus in the case $\alpha=\tau=(1+\sqrt{5}) / 2$, the golden ratio, and $b=2$, one gets the remarkable equality $\sum_{n=1}^{\infty} \frac{1}{2^{[n / \tau]}}=\left[0,2^{0}, 2^{1}, 2^{1}, 2^{2}, 2^{3}\right.$, $\left.2^{5}, \ldots\right]$, where the exponents of the partial quotients are the Fibonacci numbers.

More recently, R. L. Graham, D. E. Knuth, and O. Patashnik [8] indicated how to give a very different proof of the power series version of this result, where the number $b$ is replaced by an indeterminate (they carried out the proof for the case $\alpha=(1+\sqrt{5}) / 2)$, using the continuant polynomials of Euler [6].

In this note we give a proof, which we feel is simpler than the others, which makes use of a property of the "characteristic sequence" of $\alpha$ discovered by H. J. S. Smith [13]. The crucial idea of our approach appears in Lemma 2 below, where we regard certain initial segments of the characteristic sequence of $\alpha$ as base $b$ representations of integers.

(Böhmer, Danilov, and Adams and Davison also show that $S_{b}(\alpha)$ is transcendental for every irrational $\alpha$. We omit the proof of this fact, which is an

Received by the editors May 25, 1993 and, in revised form, November 19, 1993.

1991 Mathematics Subject Classification. Primary 11A55; Secondary 11B39. 
easy application of a theorem of Roth [11], using Lemma 3 and Theorem B below.)

Preliminaries. Let $\alpha$ be an irrational number with $0<\alpha<1$. (At the end, we will remove the restriction $\alpha<1$.) Let $\alpha=\left[0, a_{1}, a_{2}, \ldots\right]$ and $\frac{p_{n}}{q_{n}}=\left[0, a_{1}, \ldots, a_{n}\right], n \geq 0$, where $p_{n}, q_{n}$ are relatively prime non-negative integers. (As usual, we put $p_{-2}=0, p_{-1}=1, q_{-2}=1, q_{-1}=0$, so that $p_{n}=a_{n} p_{n-1}+p_{n-2}, q_{n}=a_{n} q_{n-1}+q_{n-2}$ for all $n \geq 0$.) For $n \geq 1$, define $f_{\alpha}(n)=[(n+1) \alpha]-[n \alpha]$, and consider the infinite binary sequence $f_{\alpha}=\left(f_{\alpha}(n)\right)_{n \geq 1}$, which is sometimes called the characteristic sequence of $\alpha$. Define binary words $X_{n}, n \geq 0$, by $X_{0}=0, X_{1}=0^{a_{1}-1} 1, X_{k}=X_{k-1}^{a_{k}} X_{k-2}$, $k \geq 2$, where $X^{a}$ denotes the word $X$ repeated $a$ times, and $X_{1}=1$ if $a_{1}=1$.

The following result was first proved by Smith [13]. Other proofs can be found in [2], [7], [12], and [14], and further references to the characteristic sequence can be found in [2]. Nishioka, Shiokawa, and Tamura [9] treat the more general case $[(n+1) \alpha+\beta]-[n \alpha+\beta]$.

Lemma 1. For each $n \geq 1, X_{n}$ is a prefix of $f_{\alpha}$. That is, $X_{n}=f_{\alpha}(1) f_{\alpha}(2) \ldots$ $f_{\alpha}(s)$, where $s$ is the length of $X_{n}$.

The main proof. We are now ready to prove the result stated in the Introduction. (However, we will keep the restriction $\alpha<1$ until the following section.) Let $b>1$ be an integer, let $0<\alpha<1$ be irrational, $\alpha=\left[0, a_{1}, a_{2}, \ldots\right]$, let $\frac{p_{n}}{q_{n}}=\left[0, a_{1}, \ldots, a_{n}\right], n \geq 0$, and let the binary words $X_{n}, n \geq 0$, be defined as above.

According to Lemma 1, the binary word $X_{n}$ (which has length $q_{n}$ by a trivial induction using $q_{n}=a_{n} q_{n-1}+q_{n-2}$ ) is identical with the binary word $f_{\alpha}(1) f_{\alpha}(2)$ $\cdots f_{\alpha}\left(q_{n}\right)$. If we let $x_{n}$ denote the integer whose base $b$ representation is $X_{n}$, i.e. $x_{n}=f_{\alpha}(1) b^{q_{n}-1}+f_{\alpha}(2) b^{q_{n}-2}+\cdots+f_{\alpha}\left(q_{n}\right) b^{0}$, then we can write

$$
x_{n}=b^{q_{n}} \cdot \sum_{k=1}^{q_{n}} \frac{f_{\alpha}(k)}{b^{k}} .
$$

Now we come to the crucial step.

Lemma 2. For $n \geq 0$, let $t_{n+1}=\frac{b^{q_{n+1}-b^{q_{n-1}}}}{b^{q_{n}-1}}$. Then for $n \geq 1$,

$$
x_{n+1}=t_{n+1} x_{n}+x_{n-1} \text {. }
$$

Proof. Using the facts that $X_{n}$ has length $q_{n}, X_{n-1}$ has length $q_{n-1}, x_{n+1}$ is the integer whose base $b$ representation is $X_{n+1}$, and $X_{n+1}=X_{n}^{a_{n+1}} X_{n-1}$, it follows that

$$
\begin{aligned}
x_{n+1} & =b^{q_{n-1}}\left(1+b^{q_{n}}+b^{2 q_{n}}+\cdots+b^{\left(a_{n+1}-1\right) q_{n}}\right) x_{n}+x_{n-1} \\
& =\frac{b^{q_{n-1}}\left(b^{a_{n+1} q_{n}}-1\right)}{\left(b^{q_{n}}-1\right)} x_{n}+x_{n-1}=t_{n+1} x_{n}+x_{n-1} .
\end{aligned}
$$

Lemma 3. For $n \geq 1$,

$$
\left[0, t_{1}, \ldots, t_{n}\right]=\frac{b-1}{b^{q_{n}}-1} \cdot x_{n} .
$$

Proof. Let $y_{n}=\frac{b^{q_{n}}-1}{b-1}, n \geq 0$. We show by induction on $n$ that $\left[0, t_{1}, \ldots, t_{n}\right]$ $=\frac{x_{n}}{y_{n}}$. We start the induction at $n=0$ by setting $t_{0}=0$. Note that $x_{0}=0$, 
$x_{1}=1, y_{0}=1, y_{1}=\frac{b^{q_{1}-1}}{b-1}=t_{1}$. For the induction step, we simply note that $x_{n+1}=t_{n=1} x_{n}+x_{n-1}$ and $y_{n+1}=t_{n+1} y_{n}+y_{n-1}$.

Theorem A. Let $b>1$ be an integer, and let $0<\alpha<1$ be irrational, with $f_{\alpha}(n)=[(n+1) \alpha]-[n \alpha], n \geq 1$. Let $\alpha=\left[0, a_{1}, a_{2}, \ldots\right]$, let $\frac{p_{n}}{q_{n}}=$ $\left[0, a_{1}, \ldots, a_{n}\right], n \geq 0$ (where $p_{n}, q_{n}$ are relatively prime non-negative integers), and let $t_{n}=\frac{b^{q_{n}}-b^{q_{n-2}}}{b^{q_{n-1}}-1}, n \geq 1$. Then

$$
(b-1) \sum_{k=1}^{\infty} \frac{f_{\alpha}(k)}{b^{k}}=\left[0, t_{1}, t_{2}, \ldots\right] .
$$

Proof. We have seen that $x_{n}=b^{q_{n}} \sum_{k=1}^{q_{n}} \frac{f_{\alpha}(k)}{b^{k}}$. Hence by Lemma 3,

$$
(b-1)\left(\frac{b^{q_{n}}}{b^{q_{n}}-1}\right) \sum_{k=1}^{q_{n}} \frac{f_{\alpha}(k)}{b^{k}}=\left[0, t_{1}, \ldots, t_{n}\right],
$$

and we can take the limit as $n \rightarrow \infty$.

Theorem B. With the same hypotheses as in Theorem A, we have

$$
(b-1) \sum_{n=1}^{\infty} \frac{1}{b^{[n / \alpha]}}=\left[0, t_{1}, t_{2}, \ldots\right] .
$$

Proof. This is a restatement of Theorem A, using the easily verified fact (when $0<\alpha<1)$ that $f_{\alpha}(k)=1$ if and only if $k=[n / \alpha]$ for some $n$.

Theorem $\mathrm{C}$. With the same hypotheses as in Theorem $\mathrm{A}$, we have

$$
(b-1)^{2} \sum_{k=1}^{\infty} \frac{[k \alpha]}{b^{k}}=\left[0, t_{1}, t_{2}, \ldots\right] .
$$

Proof. Using $f_{\alpha}(k)=[(k+1) \alpha]-[k \alpha]$ and $[\alpha]=0$, the series in Theorem $\mathrm{C}$ is obtained from the series in Theorem A by a slight rearrangement.

Theorem D. With the same hypotheses as in Theorem A, we have

$$
\sum_{k=1}^{\infty} \frac{f_{\alpha}(k)}{b^{k}}=(b-1) \sum_{k=1}^{\infty} \frac{(-1)^{k-1}}{\left(b^{q_{k}}-1\right)\left(b^{q_{k-1}}-1\right)} .
$$

Proof. We saw in the proof of Lemma 3 that $\left[0, t_{1}, \ldots, t_{n}\right]=\frac{x_{n}}{y_{n}}, n \geq 1$, where $y_{n}=\frac{b^{q_{n}}-1}{b-1}, n \geq 0$. By a well-known theorem (J. B. Roberts [10, p. 101]), $\frac{x_{n}}{y_{n}}=\sum_{k=1}^{n} \frac{(-1)^{k-1}}{y_{k} y_{k-1}}, n \geq 1$, and Theorem $\mathrm{D}$ now follows from Theorem A.

Removing the restriction $\alpha<1$. Now let $\alpha^{\prime}=a_{0}+\alpha$, where $a_{0} \geq 0$ is an integer, $\alpha$ is irrational, and $0<\alpha<1$.

By Theorem A we get

$$
\begin{aligned}
(b-1) \sum_{k=1}^{\infty} \frac{f_{\alpha^{\prime}}(k)}{b^{k}} & =(b-1) \sum_{k=1}^{\infty} \frac{a_{0}+f_{\alpha}(k)}{b^{k}} \\
& =(b-1) a_{0} \sum_{k=1}^{\infty} \frac{1}{b^{k}}+(b-1) \sum_{k=1}^{\infty} \frac{f_{\alpha}(k)}{b^{k}} \\
& =a_{0}+\left[0, t_{1}, t_{2}, \ldots\right]=\left[a_{0}, t_{1}, t_{2}, \ldots\right]
\end{aligned}
$$


To handle Theorem B we need to use the fact, whose simple proof we omit, that if $\alpha^{\prime}=a_{0}+\alpha$, where $0<\alpha<1$, then for each $k=0,1,2, \ldots$, the value $k$ is assumed by the expression $\left[n / \alpha^{\prime}\right]$ exactly $a_{0}+1$ times if $[n / \alpha]=k$ for some $n \geq 1$, and exactly $a_{0}$ times if $[n / \alpha]$ never equals $k$. It then follows from Theorem B that $(b-1) \sum_{n=1}^{\infty} \frac{1}{b^{\left[n / \alpha^{\prime}\right]}}=\left[a_{0} b, t_{1}, t_{2}, \ldots\right]$.

By Theorem $\mathrm{C}$ and some careful rearrangement we get $(b-1)^{2} \sum_{k=1}^{\infty} \frac{\left[k \alpha^{\prime}\right]}{b^{k}}=$ $\left[a_{0} b, t_{1}, t_{2}, \ldots\right]$.

Finally, the modified Theorem D (using the modified Theorem A) is

$$
(b-1) \sum_{k=1}^{\infty} \frac{f_{\alpha^{\prime}}(k)}{b^{k}}=a_{0}+\sum_{k=1}^{\infty} \frac{(-1)^{k-1}(b-1)^{2}}{\left(b^{q_{k}}-1\right)\left(b^{q_{k-1}}-1\right)} .
$$

Remark. This paper grew out of the first author's consideration of the number $\sum_{k=1}^{\infty} \frac{f_{\alpha}(k)}{2^{k}}$, where $\alpha=\frac{1+\sqrt{5}}{2}$, as the fixed point of the sequence $\left\{g_{n}(0)\right\}, n \geq 1$, where $g_{1}(x)=x / 2, g_{2}(x)=(x+1) / 2, g_{n}(x)=g_{n-1}\left(g_{n-2}(x)\right), n \geq 3$. This quickly leads (upon setting $g_{n}(x)=\left(x+a_{n}\right) / b_{n}$ and solving for $a_{n}$ and $b_{n}$ ) to

$$
\sum_{k=1}^{\infty} \frac{f_{\alpha}(k)}{2^{k}}=\left[0,2^{0}, 2^{1}, 2^{1}, 2^{2}, 2^{3}, 2^{5}, \ldots\right] .
$$

\section{ACKNOWLEDGMENT}

The authors are grateful to the referee for references [3] and [4] and for several helpful remarks.

\section{REFERENCES}

1. W. W. Adams and J. L. Davison, A remarkable class of continued fractions, Proc. Amer. Math. Soc. 65 (1977), 194-198.

2. T. C. Brown, Descriptions of the characteristic sequence of an irrational, Canad. Math. Bull. 36 (1993), 15-21.

3. P. E. Böhmer, Über die Transzendenz gewisser dyadischer Brüche, Math. Ann. 96 (1926), 367-377; erratum 96 (1926), 735.

4. L. V. Danilov, Some classes of transcendental numbers, Math. Notes Acad. Sci. USSR 12 (1972), 524-527.

5. J. L. Davidson, $A$ series and its associated continued fraction, Proc. Amer. Math. Soc. 63 (1977), 29-32.

6. L. Euler, Specimen algorithmi singularis, Novi Commentarii Academiae Cientiarum Petropolitanae 9 (1762), 53-69; reprinted in his Opera Omnia, Series 1, Vol. 15, pp. 31-49.

7. A. S. Fraenkel, M. Mushkin, and U. Tassa, Determination of $[n \theta]$ by its sequence of differences, Canad. Math. Bull. 21 (1978), 441-446.

8. R. L. Graham, D. E. Knuth, and O. Patashnik, Concrete mathematics, Addison-Wesley, New York, 1989.

9. K. Nishioka, I. Shiokawa, and J. Tamura, Arithmetical properties of a certain power series, J. Number Theory 42 (1992), 61-87.

10. J. B. Roberts, Elementary number theory, MIT Press, Boston, 1977.

11. K. F. Roth, Rational approximations to algebraic numbers, Mathematika 2 (1955), 1-20; corrigendum 2 (1955), 168.

12. J. Shallit, Characteristic words as fuxed points of homomorphisms, Univ. of Waterloo, Dept. of Computer Science, Tech. Report CS-91-72, 1991. 
13. H. J. S. Smith, Note on continued fractions, Messenger Math. 6 (1876), 1-14.

14. K. B. Stolarsky, Beatty sequences, continued fractions, and certain shift operators, Canad. Math. Bull. 19 (1976), 473-482.

Department of Computer Science, Rochester Institute of Technology, Rochester, NEW YORK 14623-0887

E-mail address: pga@cs.rit.edu

Department of Mathematics and Statistics, Simon Fraser University, Burnaby, Bkitish Columbia, Canada V5 A1 S6

E-mail address: tbrown@sfu.ca

Department of Mathematical Sciences, University of Nevada, Las Vegas, Nevada 89154-4020

E-mail address: shiue@nevada.edu 